\title{
Erratum: Unified view of quantum correlations and quantum coherence [Phys. Rev. A 94, 022329 (2016)]
}

\author{
Kok Chuan Tan, Hyukjoon Kwon, Chae-Yeun Park, and Hyunseok Jeong \\ (Received 22 November 2017; published 14 December 2017)
}

DOI: 10.1103/PhysRevA.96.069905

It was brought to our attention that there exists a counterexample to the claims of Proposition 1 of our paper. We verified that the counterexample is indeed correct, and so the claims of Proposition 1 are in error. The proposition is intended to be supplemental in nature and does not impact the key results presented in our paper, which discusses the procedures that will identify quantum discord and entanglement through coherence-based quantities.

The counterexample is as follows. Consider the bipartite state,

$$
\rho=\frac{1}{2}\left(|+\rangle_{A}\langle+|\otimes| 0\rangle_{B}\langle 0|+|-\rangle_{A}\langle-|\otimes| 1\rangle_{B}\langle 1|\right) .
$$

This state clearly has nonzero coherence with respect to the local bases $\{|0\rangle,|1\rangle\}$ on both parties. A projection onto this local basis on the subsystem $B$ will leave the state unchanged, so $\sum_{i=0,1}|i\rangle_{B}\langle i|\rho| i\rangle_{B}\langle i|=\rho$.

However, a projection onto the $\{|+\rangle,|-\rangle\}$ basis where $| \pm\rangle:=\frac{1}{\sqrt{2}}(|0\rangle \pm|1\rangle)$ results in the following:

$$
\sum_{j=+,-}|j\rangle_{B}\langle j|\rho| j\rangle_{B}\langle j|=\frac{1}{4} \mathbb{1}_{A B} .
$$

The above state is clearly diagonal and so contains zero coherence. In this example, it is then clear that the projection onto the local basis is not the projection that induces the greatest coherence loss. The claims of Proposition 1 are therefore not correct.

Nevertheless, one may still observe that for a pure state, there is always a local eigenbasis that induces maximal coherence loss. To see this, we note that any bipartite state can be written in terms of its Schmidt decomposition of a bipartite state,

$$
|\psi\rangle=\sum_{i} \sqrt{p_{i}}|i\rangle_{A}|i\rangle_{B}
$$

It is then immediately seen that the reduced density matrices are $\rho_{A}=\sum_{i} p_{i}|i\rangle_{A}\langle i|$ and $\rho_{B}=\sum_{i} p_{i}|i\rangle_{B}\langle i|$, so $\left\{|i\rangle_{A}\right\}$ and $\left\{|i\rangle_{B}\right\}$ are a set of eigenstates, and hence form a set of local eigenbases for both parties. A projection onto one of these local eigenbases results in the diagonal state,

$$
\sum_{i}|i\rangle_{B}\langle i|(|\psi\rangle\langle\psi|)| i\rangle_{B}\left\langle i\left|=\sum_{i} p_{i}\right| i\right\rangle_{A}\langle i|\otimes| i\rangle_{B}\langle i|,
$$

so we see that with respect to the local eigenbases $\left\{|i\rangle_{A}\right\}$ and $\left\{|i\rangle_{B}\right\}$, the projection completely decoheres the state and so is maximally decohering. Indeed, for the rest of our paper, the focus of the discussion revolves around the local eigenbases, and not the general local bases which Proposition 1 considers. As before, this observation provides additional intuition for the reader with regards to the effects of local projections on the coherence of the total state, but is not necessary to follow the rest of the results in our paper.

We thank Y. Yao, L. Ge, and M. Li for pointing out this counterexample to us. 